\title{
ON SPECIAL GENERIC MAPS INTO $\mathbf{R}^{3}$
}

\author{
Osamu Saeki and Kazuhiro Sakuma
}

\begin{abstract}
Let $f: M \rightarrow \mathbf{R}^{p}$ be a smooth map of a closed $n$-dimensional manifold $M$ into $\mathbf{R}^{p}(n \geq p)$ which has only definite fold singularities as its singular points. Such a map is called a special generic map, which was first defined by Burlet and de Rham for $(n, p)=(3,2)$ and later extended to general $(n, p)$ by Porto, Furuya, Sakuma and Saeki. In this paper, we study the global topology of such maps for $p=3$ and give various new results, among which are a splitting theorem for manifolds admitting special generic maps into $R^{3}$ and a classification theorem of 4and 5-dimensional manifolds with free fundamental groups admitting special generic maps into $\mathbf{R}^{3}$. Furthermore, we study the topological structure of the surfaces which arise as the singular set of a special generic map into $R^{3}$ on a given manifold.
\end{abstract}

\section{Introduction.}

A special generic map is a smooth map of a closed $n$-dimensional manifold into $\mathbf{R}^{p}(n \geq p)$ all of whose singularities are the definite fold points. This class of maps has been first studied by Burlet and de Rham [4], who have classified those closed 3-dimensional manifolds which admit special generic maps into $\mathbf{R}^{2}$. This result has been generalized to manifolds of arbitrary dimensions which admit special generic maps into $\mathbf{R}^{2}$ by Porto and Furuya [30] and Saeki [31]. These results imply that if a manifold of dimension greater than or equal to three admits a special generic map into $\mathbf{R}^{2}$, then its fundamental group must be a free group. Note also that Sakuma [34] and Saeki [31], [32] have studied special generic maps of simply connected manifolds into $\mathbf{R}^{3}$. In this paper, we consider special generic maps of nonsimply connected manifolds into $\mathbf{R}^{3}$ in general and give various new results about the global topology of such maps. We will see that the fundamental group of a manifold admitting a special generic map into $\mathbf{R}^{3}$ is not a free group any more in general, but that it behaves very much like a 3-manifold group.

In $\S 2$, we consider special generic maps of a manifold whose fundamental group is decomposed into a nontrivial free product. We show that then 
the manifold splits into a connected sum of two nonsimply connected manifolds with corresponding fundamental groups both of which admit a special generic map into $\mathbf{R}^{3}$. This corresponds to Kneser's conjecture for 3dimensional manifolds (see, for example, [19]). Note that this type of splitting theorem does not hold in general for higher dimensional manifolds (see $[5],[6],[25])$.

In $\S 3$, using the above splitting theorem, we classify those 4- and 5dimensional manifolds with free fundamental groups which admit special generic maps into $\mathbf{R}^{3}$. This is a generalization of results in [34], [31], [32], where simply connected manifolds admitting special generic maps into $\mathbf{R}^{3}$ have been studied. Using this classification theorem, we give some examples of pairs of homeomorphic smooth 4-manifolds $\left(M_{1}, M_{2}\right)$ such that $M_{1}$ admits a special generic map into $\mathbf{R}^{3}$ while $M_{2}$ does not. Such an example has been first given in [32], where the manifolds are simply connected. Here we give examples of nonorientable manifolds with infinite cyclic fundamental groups, using constructions due to Akbulut [1], [2], [3] and Kreck [23]. These examples show that the existence of a special generic map on a given manifold is strongly related to its smooth structure; in other words, from the singularity theoretical viewpoint, the existence of an exotic smooth structure is closely related to the obstruction to the elimination of singularities other than the definite fold ones (see [33]).

In $\S 4$, we study the fundamental groups of manifolds admitting special generic maps into $\mathbf{R}^{3}$. Here we consider the quotient space (or the Stein factorization) $W_{f}$ of a special generic map $f: M \rightarrow \mathbf{R}^{3}$. It is defined to be the space of all connected components of the fibers of $f$ and it is known that it has a structure of a compact orientable 3-manifold with fundamental group isomorphic to that of $M$ [31]. Then we can obtain various properties on $\pi_{1}(M)$ using results on 3-manifold groups [19]. In some cases, we can even specify the diffeomorphism type of the source manifold. For example, we show that if $\pi_{1}(M)$ is a surface group and $\operatorname{dim} M=4,5,6$ or 7 , then $M$ is diffeomorphic to the connected sum of a smooth $S^{n-2}$-bundle over a surface, some smooth $S^{n-2}$-bundles over $S^{2}$ and a homotopy $n$-sphere (Theorem 4.3).

In $\S 5$, we give a necessary condition on the surfaces which arise as the singular set of a special generic map into $\mathbf{R}^{3}$ on a given manifold $M$. More precisely, when the fundamental group of $M$ is indecomposable with respect to free products, then the number of sphere components of the singular set is an invariant of $M$; in fact, it is determined by the $L^{2}$-Betti numbers of $M$ (for the definition and some properties of the $L^{2}$-Betti numbers, see [27], [28], [17], for example).

In $\S 6$, we show that for an arbitrary pair of closed orientable (not necessarily connected) surfaces $\left(S_{1}, S_{2}\right)$, there exists a smooth closed 4-dimensional 
manifold $M$ which admits special generic maps $f_{1}, f_{2}: M \rightarrow \mathbf{R}^{3}$ whose singular sets $S\left(f_{1}\right)$ and $S\left(f_{2}\right)$ are diffeomorphic to $S_{1}$ and $S_{2}$ respectively. In this case, the fundamental group of $M$ is decomposable in general. Furthermore we pose some questions concerning the number of equivalence classes of special generic maps on a given manifold.

Throughout the paper, manifolds and maps are of class $C^{\infty}$ and the sym-

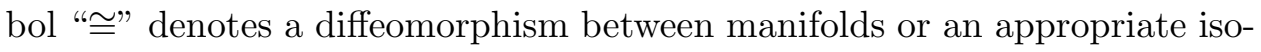
morphism between algebraic objects.

The authors would like to express their sincere gratitude to Mahito Kobayashi and Yasuhiro Hara for stimulating discussions. The authors also would like to thank the referee for his/her useful comments and suggestions.

\section{A Kneser type splitting theorem.}

In this section, we prove the following.

Theorem 2.1. Let $f: M \rightarrow \mathbf{R}^{3}$ be a special generic map of a closed connected $n$-dimensional manifold. Suppose that $\pi_{1}(M)$ is isomorphic to a nontrivial free product $\Gamma_{1} * \Gamma_{2}$. If $n=4,5$ or 6 , then there exist two closed $n$-dimensional manifolds $M_{1}$ and $M_{2}$ such that $M$ is diffeomorphic to the connected sum $M_{1} \sharp M_{2}$, that $\pi_{1}\left(M_{i}\right)$ is isomorphic to $\Gamma_{i}(i=1,2)$ and that there exist special generic maps $f_{i}: M_{i} \rightarrow \mathbf{R}^{3}(i=1,2)$.

Proof. Consider the quotient map $q_{f}: M \rightarrow W_{f}$ in the Stein factorization of $f: M \rightarrow \mathbf{R}^{3}$ [31] (see also [4] or [30]). Note that $W_{f}$ is a compact connected orientable 3 -manifold with nonempty boundary $\partial W_{f}$. Set $\partial W_{f}=$ $F_{1} \cup \cdots \cup F_{r}$, where $F_{j}$ are the connected components.

First suppose that all the components $F_{j}$ are incompressible in $W_{f}$. In this case, by Kneser's conjecture (for example, see [19, Chapter 7]), there exist compact connected 3-manifolds $W_{1}^{\prime}$ and $W_{2}^{\prime}$ such that $W_{f}$ is diffeomorphic to the connected sum $W_{1}^{\prime} \sharp W_{2}^{\prime}$ and that $\pi_{1}\left(W_{i}^{\prime}\right)$ is isomorphic to $\Gamma_{i}(i=1,2)$. Since $\partial W_{f}$ is nonempty, we may assume that $\partial W_{1}^{\prime} \neq \emptyset$. Set $W_{1}=W_{1}^{\prime}$ and $W_{2}=W_{2}^{\prime}-\operatorname{Int} D^{3}$, where $D^{3}$ is a 3 -disk embedded in the interior of $W_{2}^{\prime}$. Then it is easy to see that $W_{1}^{\prime} \sharp W_{2}^{\prime}$ is diffeomorphic to the boundary connected sum $W_{1} \curvearrowleft W_{2}$. Set $B=W_{1} \cap W_{2}$, which is a properly embedded 2-disk in $W_{f}$. We may assume that $B$ is transverse to $\partial W_{f}$. Then we see easily that $\tilde{B}=q_{f}^{-1}(B)$ is an $(n-1)$-dimensional submanifold of $M$ which admits a Morse function with exactly two critical points. Since $n-1=3,4$ or 5 by our assumption, we see that $\tilde{B}$ is diffeomorphic to the standard $(n-1)$-sphere. Set $M_{i}^{\prime}=q_{f}^{-1}\left(W_{i}\right)$ and set $M_{i}=M_{i}^{\prime} \cup D^{n}(i=1,2)$, where we attach the $n$-dimensional disk $D^{n}$ along the boundary. Note that $M_{i}$ are smooth closed $n$-dimensional manifolds. Then we see that $M$ is 
diffeomorphic to the connected sum $M_{1} \sharp M_{2}$. In order to construct special generic maps $f_{i}: M_{i} \rightarrow \mathbf{R}^{3}$, we need the following.

Lemma 2.2. Let $f, g: S^{n} \rightarrow \mathbf{R}^{2}$ be special generic maps of the standard $n$-dimensional sphere $S^{n}$. If $n=3,4$ or 5 , then there exist diffeomorphisms $H: S^{n} \rightarrow S^{n}$ and $h: W_{f} \rightarrow W_{g}$ such that $q_{g} \circ H=h \circ q_{f}$.

Proof. By [31], $W_{f}$ and $W_{g}$ are diffeomorphic to the 2-dimensional disk. Let $C_{f}$ (resp. $C_{g}$ ) be a closed collar neighborhood of $\partial W_{f}$ (resp. $\partial W_{g}$ ) in $W_{f}$ (resp. in $\left.W_{g}\right)$. Set $M_{f}=q_{f}^{-1}\left(\overline{W_{f}-C_{f}}\right)$ and $M_{g}=q_{g}^{-1}\left(\overline{W_{g}-C_{g}}\right)$. Since $\left.q_{f}\right|_{M_{f}}$ and $\left.q_{g}\right|_{M_{g}}$ are projections of smooth $S^{n-2}$-bundles over $\overline{W_{f}-C_{f}}$ $\left(\cong D^{2}\right)$ and $\overline{W_{g}-C_{g}}\left(\cong D^{2}\right)$ respectively, there exist diffeomorphisms $H_{1}$ : $M_{f} \rightarrow M_{g}$ and $h_{1}: \overline{W_{f}-C_{f}} \rightarrow \overline{W_{g}-C_{g}}$ such that $q_{g} \circ H_{1}=h_{1} \circ q_{f}$ on $M_{f}$. Let $\pi_{f}: C_{f} \rightarrow \partial W_{f}$ and $\pi_{g}: C_{g} \rightarrow \partial W_{g}$ be natural projections and set $N_{f}=q_{f}^{-1}\left(C_{f}\right)$ and $N_{g}=q_{f}^{-1}\left(C_{g}\right)$. Then by [31], $\left.\pi_{f} \circ q_{f}\right|_{N_{f}}$ and $\left.\pi_{g} \circ q_{g}\right|_{N_{g}}$ are projections of linear $D^{n-1}$-bundles over $\partial W_{f}$ and $\partial W_{g}$ respectively. (Here a bundle is called linear if its structure group is reduced to an orthogonal group.) Since they are orientable, they are necessarily trivial. Recall that the natural inclusion $S O(n-1) \rightarrow \operatorname{Diff}^{+}\left(S^{n-2}\right)$ induces a homotopy equivalence for $n=3,4$ and 5 [9], [38], [18], where $\operatorname{Diff}^{+}\left(S^{n-2}\right)$ denotes the group of orientation preserving diffeomorphisms of $S^{n-2}$. Then we see that the diffeomorphisms $H_{1}: M_{f} \rightarrow M_{g}$ and $h_{1}: \overline{W_{f}-C_{f}} \rightarrow \overline{W_{g}-C_{g}}$ extend to diffeomorphisms $H: M_{f} \cup N_{f} \rightarrow M_{g} \cup N_{g}$ and $h: \overline{W_{f}-C_{f}} \cup C_{f} \rightarrow$ $\overline{W_{g}-C_{g}} \cup C_{g}$ respectively with the desired properties. This completes the proof.

Using the above lemma, we can easily construct special generic maps $f_{i}: M_{i} \rightarrow \mathbf{R}^{3}$ whose quotient spaces $W_{f_{i}}$ are diffeomorphic to $W_{i}(i=1,2)$ (for example, see the argument in the proof of [31, Lemma 5.4]). Then by [31, Proposition 3.9], $\pi_{1}\left(M_{i}\right)$ is isomorphic to $\pi_{1}\left(W_{i}\right) \cong \Gamma_{i}$.

Now suppose that $F_{j}$ is not incompressible for some $j$. Then by Dehn's lemma, there exists a properly embedded 2-disk $B$ in $W_{f}$ such that $c=\partial B$ is an essential simple closed curve in $F_{j}$.

Case 1. When $c$ does not separate $F_{j}$.

Let $N$ be a regular neighborhood of $B$ in $W_{f}$ and set $W_{1}^{\prime}=\overline{W_{f}-N}$. Since $c$ does not separate $F_{j}$, the two 2-disks $W_{1}^{\prime} \cap N$ lie in the same component of $\partial W_{1}^{\prime}$. Then it is easy to see that $W_{f}$ is diffeomorphic to the boundary connected sum $W_{1}^{\prime} \downarrow W_{2}^{\prime}$, where $W_{2}^{\prime}$ is diffeomorphic to $S^{1} \times D^{2}$. Consequently $\pi_{1}\left(W_{f}\right)$ is isomorphic to the free product $\pi_{1}\left(W_{1}^{\prime}\right) * \mathbf{Z}$. Furthermore, by an argument similar to the above, we see that there exist two closed $n$-dimensional manifolds $M_{1}^{\prime}$ and $M_{2}^{\prime}$ such that $M$ is diffeomorphic to the connected sum 
$M_{1}^{\prime} \sharp M_{2}^{\prime}$, that $\pi_{1}\left(M_{i}^{\prime}\right)$ is isomorphic to $\pi_{1}\left(W_{i}^{\prime}\right)$ and that there exist special generic maps $f_{i}^{\prime}: M_{i}^{\prime} \rightarrow \mathbf{R}^{3}$ with $W_{f_{i}^{\prime}}$ diffeomorphic to $W_{i}^{\prime}(i=1,2)$.

Case 2. When $c$ separates $F_{j}$ and $B$ does not separate $W_{f}$.

In this case, using the same notation as above, we see that $W_{f}$ is obtained from $W_{1}^{\prime}$ by attaching a 1-handle $N$ to distinct boundary components of $W_{1}^{\prime}$. Set $M_{1}^{\prime}=q_{f}^{-1}\left(W_{1}^{\prime}\right)$ and $M_{2}^{\prime}=q_{f}^{-1}(N)$. Then we see that $\partial M_{1}^{\prime}$ consists of two $(n-1)$-spheres and that $M_{2}^{\prime}$ is diffeomorphic to $S^{n-1} \times[-1,1]$. Set $M_{1}^{\prime \prime}=M_{1}^{\prime} \cup D^{n} \cup D^{n}$, where we attach the two $n$-dimensional disks along the boundaries. Then it is easy to see that $M$ is diffeomorphic to the connected sum $M_{1}^{\prime \prime} \sharp M_{2}^{\prime \prime}$, where $M_{2}^{\prime \prime}$ is diffeomorphic to $S^{1} \times S^{n-1} \sharp \Sigma$ and $\Sigma$ is a homotopy $n$-sphere which admits a Morse function with exactly two critical points. Since $n=4,5$ or $6, \Sigma$ is diffeomorphic to the standard $n$-sphere. Furthermore, we can construct a special generic map $f_{1}^{\prime \prime}: M_{1}^{\prime \prime} \rightarrow \mathbf{R}^{3}$ such that $W_{f_{1}^{\prime \prime}}$ is diffeomorphic to $W_{1}^{\prime}$. We can also construct a special generic map $f_{2}^{\prime \prime}: M_{2}^{\prime \prime} \rightarrow \mathbf{R}^{3}$, since $M_{2}^{\prime \prime}$ is diffeomorphic to $S^{1} \times S^{n-1}$ (see [32, (5.3.5)]). Note that $\pi_{1}(M)$ is isomorphic to the free product $\pi_{1}\left(W_{1}^{\prime}\right) * \mathbf{Z}$.

Case 3. When $B$ separates $W_{f}$.

In this case, using the above notation, $\overline{W_{f}-N}$ consists of two components $W_{1}^{\prime}$ and $W_{2}^{\prime}$. Since $c$ is essential in $F_{j}$, both $W_{1}^{\prime}$ and $W_{2}^{\prime}$ have non-spherical boundary components, and hence their fundamental groups are nontrivial. Furthermore it is easy to see that $W_{f}$ is diffeomorphic to the boundary connected sum $W_{1}^{\prime} \downarrow W_{2}^{\prime}$. Then by using an argument similar to the above, we see that there exist two closed $n$-dimensional manifolds $M_{1}^{\prime}$ and $M_{2}^{\prime}$ such that $M$ is diffeomorphic to the connected sum $M_{1}^{\prime} \sharp M_{2}^{\prime}$, that there exist special generic maps of $M_{i}^{\prime}$ into $\mathbf{R}^{3}(i=1,2)$, and that $\pi_{1}\left(M_{i}^{\prime}\right)$ is isomorphic to $\pi_{1}\left(W_{i}^{\prime}\right)(i=1,2)$.

Recall that the finitely generated group $\pi_{1}(M)$ has a free product decomposition $G_{1} * \cdots * G_{s}$ with each $G_{j}$ being indecomposable and that the indecomposable factors are unique up to order and isomorphism by the Kurosh subgroup theorem (for example, see [19, Chapter 8]). By iterating the above arguments, we see that there exist closed connected $n$-dimensional manifolds $\tilde{M}_{j}(j=1,2, \ldots, s)$ such that $M$ is diffeomorphic to the connected sum $\tilde{M}_{1} \sharp \cdots \sharp \tilde{M}_{s}$, that $\pi_{1}\left(\tilde{M}_{j}\right)$ is isomorphic to $G_{j}$ and that there exist special generic maps $\tilde{f}_{j}: \tilde{M}_{j} \rightarrow \mathbf{R}^{3}(j=1,2, \ldots, s)$. Then the theorem follows easily. This completes the proof.

Compare Theorem 2.1 with [5], [6], [25]. In particular, the 4-manifold $M$ in $[\mathbf{2 5}$, Theorem 0.2$]$ does not admit any special generic map into $\mathbf{R}^{3}$. 
Remark 2.3. Note that a uniqueness in the strong sense does not hold in Theorem 2.1. For example, $M=\left(S^{1} \times S^{3}\right) \sharp\left(T^{2} \times S^{2}\right) \sharp\left(S^{2} \times S^{2}\right)$ admits a special generic map into $\mathbf{R}^{3}$. The fundamental group $\pi_{1}(M)$ decomposes as the free product $\mathbf{Z} *(\mathbf{Z} \times \mathbf{Z})$ and $M$ has two corresponding decompositions $M_{1} \sharp M_{2}$ and $M_{1}^{\prime} \sharp M_{2}^{\prime}$, where $M_{1}=S^{1} \times S^{3}, M_{2}=\left(T^{2} \times S^{2}\right) \sharp\left(S^{2} \times S^{2}\right), M_{1}^{\prime}=$ $\left(S^{1} \times S^{3}\right) \sharp\left(S^{2} \times S^{2}\right)$ and $M_{2}^{\prime}=T^{2} \times S^{2}$. We do not know if a "uniqueness" in a weaker sense holds in Theorem 2.1. Note that, for manifolds of dimension four, a "stable uniqueness" for Kneser type splittings of a given manifold is proved in [24] without any consideration on special generic maps on the manifold.

Remark 2.4. If we ignore the existence of special generic maps on the connected sum factors, we can obtain a similar splitting theorem for all $n$ with $\Theta_{n-1}=0$, where $\Theta_{m}$ is the $h$-cobordism group of oriented homotopy $m$-spheres. For example, this is valid for $n=7,13$. Furthermore, when the manifold $M$ is stably parallelizable, the same splitting theorem is valid for all $n$ with $\Theta_{n-1}(\partial \pi)=0$, where $\Theta_{m}(\partial \pi)$ is the $h$-cobordism group of oriented homotopy $m$-spheres which bound compact parallelizable $(m+1)$ dimensional manifolds. Note that $\Theta_{n-1}(\partial \pi)=0$ if $n(\geq 7)$ is odd or $n=14$.

Problem 2.5. Is the quasi-equivalence class of special generic maps of $S^{n}$ $(n \geq 3)$ into $\mathbf{R}^{2}$ unique? (Here, two special generic maps are said to be quasi-equivalent (see [32]) if their quotient maps are right-left equivalent in the sense of Lemma 2.2.) Note that this is true for $n=3,4$ and 5 by Lemma 2.2.

\section{Manifolds with free fundamental groups admitting a special generic map into $\mathbf{R}^{3}$.}

In this section, we first give a complete list of 4- and 5-dimensional manifolds with free fundamental groups admitting a special generic map into $\mathbf{R}^{3}$. Using this result, we will see that the existence of a special generic map on a given manifold is strongly related to the smooth structure especially for 4dimensional manifolds.

First, we prove the following.

Theorem 3.1. Let $M$ be a closed connected $n$-dimensional manifold with free fundamental group. We suppose that $n=4$ or 5 . Then $M$ admits a special generic map into $\mathbf{R}^{3}$ if and only if $M$ is diffeomorphic to

$$
\left(\sharp^{r-\varepsilon} S^{1} \times S^{n-1}\right) \sharp\left(\sharp^{\varepsilon} S^{1} \tilde{\times} S^{n-1}\right) \sharp\left(\sharp^{s} S^{2} \times S^{n-2}\right) \sharp\left(\sharp^{\delta} S^{2} \tilde{x} S^{n-2}\right) \sharp \Sigma^{n}
$$


for some $\varepsilon, \delta \in\{0,1\}$ and $s \geq 0$, where $r$ is the rank of the free group $\pi_{1}(M)$, the connected sum over the empty set is assumed to be the standard $n$-sphere, $S^{1} \tilde{\times} S^{n-1}$ is the nonorientable $S^{n-1}$-bundle over $S^{1}, S^{2} \tilde{\times} S^{n-2}$ is the nontrivial $S^{n-2}$-bundle over $S^{2}, \Sigma^{n}$ is the standard $n$-sphere for $n=5$, and $\Sigma^{n}=\partial\left(\Delta \times D^{2}\right)$ for some compact contractible 3 -manifold $\Delta$ for $n=4$.

Note that the above theorem for the trivial fundamental group has been proved in [31] and [32] (see also [34]). Furthermore, in the case where $M$ is orientable of dimension four and the quotient space $W_{f}$ is homeomorphic to a boundary connected sum of some solid tori, a similar result has been obtained in [20].

Proof of Theorem 3.1. It is not difficult to see that the manifolds in the above list admit a special generic map into $\mathbf{R}^{3}$ (see [31], [32]).

Conversely, suppose that $M$ admits a special generic map $f$ into $\mathbf{R}^{3}$. In view of Theorem 2.1 together with the facts that $S^{1} \tilde{\times} S^{n-1} \sharp S^{1} \tilde{\times} S^{n-1} \cong$ $S^{1} \times S^{n-1} \sharp S^{1} \tilde{\times} S^{n-1}$ and $S^{2} \tilde{\times} S^{n-2} \sharp S^{2} \tilde{\times} S^{n-2} \cong S^{2} \times S^{n-2} \sharp S^{2} \tilde{\times} S^{n-2} \quad([\mathbf{3 2}$, Lemma 2.2]), we may assume that $\pi_{1}(M)$ is the infinite cyclic group. Then by [19, Chapter 5], we see that $W_{f}$ is diffeomorphic to $\Sigma \sharp B_{1} \sharp \cdots \sharp B_{s} \sharp S^{1} \times D^{2}$ or $\Sigma \sharp B_{1} \sharp \cdots \sharp B_{t} \sharp S^{1} \times S^{2}$, where $\Sigma$ is a homotopy 3 -sphere, $B_{i}$ are the 3 dimensional disks, $s \geq 0$ and $t \geq 1$. Thus $W_{f}$ is diffeomorphic to $\Delta \natural\left(\natural^{s}\left(S^{2} \times\right.\right.$ $I)) \llbracket S^{1} \times D^{2}$ or $\Delta \natural\left(\natural^{t-1}\left(S^{2} \times I\right)\right) \natural\left(S^{1} \times S^{2}-\right.$ Int $\left.D^{3}\right)$ respectively, where $I=[0,1]$ and $\Delta=\Sigma-\operatorname{Int} D^{3}$ is a compact contractible 3 -manifold. Corresponding to the above decomposition of $W_{f}$, we have a connected sum decomposition $M=M_{1} \sharp \cdots \sharp M_{r}$ as in the proof of Theorem 2.1, where $r=s+2$ or $t+1$ and each $M_{i}$ admits a special generic map into $\mathbf{R}^{3}$ whose quotient space $W_{i}$ is diffeomorphic to $\Delta, S^{2} \times I, S^{1} \times D^{2}$ or $S^{1} \times S^{2}-\operatorname{Int} D^{3}$. If $W_{i} \cong \Delta$, then $M_{i} \cong \partial\left(\Delta \times D^{n-2}\right)$, which is diffeomorphic to the standard 5-sphere when $n=5$. If $W_{i} \cong S^{2} \times I$, then $M_{i}$ is diffeomorphic to an $S^{n-2}$-bundle over $S^{2}$, which is diffeomorphic to $S^{2} \times S^{n-2}$ or $S^{2} \tilde{\times} S^{n-2}$. If $W_{i} \cong S^{1} \times D^{2}$, then $M_{i}$ is diffeomorphic to an $S^{n-1}$-bundle over $S^{1}$, which is diffeomorphic to $S^{1} \times S^{n-1}$ or $S^{1} \tilde{x} S^{n-1}$. Finally, if $W_{i} \cong S^{1} \times S^{2}-\operatorname{Int} D^{3}$, then by using an argument similar to that used in Case 2 of the proof of Theorem 2.1, we can show that $M_{i}$ is diffeomorphic to the connected sum $M_{i, 1} \sharp M_{i, 2}$, where $M_{i, 1}$ admits a special generic map into $\mathbf{R}^{3}$ whose quotient space is diffeomorphic to $S^{2} \times I$ and $M_{i, 2}$ is diffeomorphic to an $S^{n-1}$-bundle over $S^{1}$. This completes the proof.

Compare Theorem 3.1 with [8] for $n=4$. 
Remark 3.2. When $n=6$ or 7 , we can show that if $M$ admits a special generic map into $\mathbf{R}^{3}$ with $\pi_{1}(M)$ free, then $M$ is diffeomorphic to $M_{1} \sharp \cdots \sharp M_{r} \sharp N_{1} \sharp \cdots \sharp N_{s} \sharp \Sigma^{n}$, where $M_{i}$ is a smooth $S^{n-1}$-bundle over $S^{1}, N_{j}$ is a smooth $S^{n-2}$-bundle over $S^{2}$ and $\Sigma^{n}$ is a homotopy $n$-sphere.

Problem 3.3. (1) Are there no repetitions in the list of Theorem 3.1 when $n=5$ ? (Compare this with the result in [32] for the simply connected case.)

(2) Classify the special generic maps as in Theorem 3.1 up to regular equivalence or up to quasi-equivalence (see [32]).

(3) Is $\Sigma^{4} \sharp S^{1} \times S^{3}$ (resp. $\Sigma^{4} \sharp S^{1} \tilde{\times} S^{3}$ ) diffeomorphic to $S^{1} \times S^{3}$ (resp. $S^{1} \tilde{\times} S^{3}$ ) in Theorem 3.1? See [31, Remark 6.5] or [35]. Note that $\Sigma^{4} \sharp S^{1} \times S^{3}$ is diffeomorphic to $S^{1} \times S^{3}$ if and only if $\Sigma^{4}$ is diffeomorphic to $S^{4}$ (see $[22$, Proposition]).

In the following, we give some examples of pairs of homeomorphic smooth 4-manifolds $\left(M_{1}, M_{2}\right)$ with the infinite cyclic fundamental group such that $M_{1}$ admits a special generic map into $\mathbf{R}^{3}$ while $M_{2}$ does not. Such an example for simply connected 4-manifolds has already been given in [32].

First, we recall the construction of the Akbulut manifold ([1], [2], [3], [14]). Let $Q^{4}$ be a Cappell-Shaneson's exotic $\mathbf{R} P^{4}$ (see [7]). Recall that $Q^{4}$ is homeomorphic to $\mathbf{R} P^{4}([\mathbf{1 5}])$ and that $Q^{4} \sharp\left(\sharp^{k} S^{2} \times S^{2}\right)$ is not smoothly $h$ cobordant to $\mathbf{R} P^{4} \sharp\left(\sharp^{k} S^{2} \times S^{2}\right)$ for any nonnegative integer $k$ ([7]). Akbulut [1] has found an embedding $\varphi: \mathbf{R} P^{2} \hookrightarrow Q^{4} \sharp S^{2} \times S^{2}$ such that $\pi_{1}\left(Q^{4} \sharp S^{2} \times\right.$ $\left.S^{2}-\varphi\left(\mathbf{R} P^{2}\right)\right) \cong \mathbf{Z}$ and that the normal bundle of $\varphi$ is isomorphic to that of a standardly embedded $\mathbf{R} P^{2}$ in $\mathbf{R} P^{4}$. Then we set $M=\left(Q^{4} \sharp S^{2} \times S^{2}-\right.$ Int $N) \cup S^{1} \tilde{\times} D^{3}$, where $N$ is a closed tubular neighborhood of $\varphi\left(\mathbf{R} P^{2}\right)$ in $Q^{4} \sharp S^{2} \times S^{2}$ and $S^{1} \tilde{\times} D^{3}$ is the nontrivial (and hence nonorientable) $D^{3}$ bundle over $S^{1}$. This is the so-called Akbulut manifold and it is known to be an exotic $S^{1} \tilde{\times} S^{3} \sharp S^{2} \times S^{2}$ (see [1], [2], [3], [14]). Then we have the following.

Theorem 3.4. Let $M$ be the Akbulut manifold. Then for every nonnegative integer $k, M \sharp\left(\sharp^{k} S^{2} \times S^{2}\right)$ is homeomorphic to $S^{1} \tilde{\times} S^{3} \sharp\left(\sharp^{k+1} S^{2} \times S^{2}\right)$, while $M \sharp\left(\sharp^{k} S^{2} \times S^{2}\right)$ does not admit any special generic map into $\mathbf{R}^{3}$.

Proof. It is known that $M$ is topologically s-cobordant to $S^{1} \tilde{\times} S^{3} \sharp S^{2} \times S^{2}$ (cf. [39, p. 159]) and hence by Freedman [15] it is homeomorphic to $S^{1} \tilde{\times} S^{3} \sharp S^{2} \times$ $S^{2}$. Therefore we see that for every nonnegative integer $k, M \sharp\left(\sharp^{k} S^{2} \times S^{2}\right)$ is homeomorphic to $S^{1} \tilde{\times} S^{3} \sharp\left(\sharp^{k+1} S^{2} \times S^{2}\right)$.

Suppose that $M \sharp\left(\sharp^{k} S^{2} \times S^{2}\right)$ were diffeomorphic to $\Sigma \sharp S^{1} \tilde{\times} S^{3} \sharp\left(\sharp^{k+1} S^{2} \times S^{2}\right)$ for some homotopy 4 -sphere $\Sigma$. Then it is easily seen that $M \sharp\left(\sharp{ }^{k} S^{2} \times S^{2}\right)$ and $S^{1} \tilde{\times} S^{3} \sharp\left(\sharp^{k+1} S^{2} \times S^{2}\right)$ are smoothly $s$-cobordant, since every homotopy 4 -sphere is $h$-cobordant to the standard 4 -sphere. However, this contradicts 
a result in [3]; in fact, they differ by nontrivial normal invariant. Thus $M \sharp\left(\sharp^{k} S^{2} \times S^{2}\right)$ is not diffeomorphic to $\Sigma \sharp S^{1} \tilde{\times} S^{3} \sharp\left(\sharp^{k+1} S^{2} \times S^{2}\right)$ for any homotopy 4 -sphere $\Sigma$. Then by Theorem 3.1, we see that $M$ does not admit a special generic map into $\mathbf{R}^{3}$. This completes the proof.

Remark 3.5. We note that for the Akbulut manifold $M, M \sharp S^{2} \tilde{\times} S^{2}$ is diffeomorphic to $S^{1} \tilde{\times} S^{3} \sharp S^{2} \times S^{2} \sharp S^{2} \tilde{\times} S^{2}$ (see [2], [3]) and hence it admits a special generic map into $\mathbf{R}^{3}$. Furthermore, the double cover $\tilde{M}$ of $M$ is diffeomorphic to $S^{1} \times S^{3} \sharp\left(\sharp^{2} S^{2} \times S^{2}\right)$ [14] and hence admits a special generic map into $\mathbf{R}^{3}$. The first fact shows that the following statement is not true in general: for closed 4-dimensional manifolds $M_{1}$ and $M_{2}$, if $M_{1}$ and $M_{1} \sharp M_{2}$ admit special generic maps into $\mathbf{R}^{3}$, then so does $M_{2}$.

We give another example as follows.

Theorem 3.6. Let $K$ be a $K 3$ surface. Then for every integer $r$ with $r \geq 0, S^{1} \tilde{\times} S^{3} \sharp K \sharp\left(\sharp^{r} S^{2} \times S^{2}\right)$ is homeomorphic to $S^{1} \tilde{\times} S^{3} \sharp\left(\sharp^{r+11} S^{2} \times S^{2}\right)$, while $S^{1} \tilde{\times} S^{3} \sharp K \sharp\left(\sharp^{r} S^{2} \times S^{2}\right)$ does not admit any special generic map into $\mathbf{R}^{3}$.

Proof. As shown in [23, Lemma 1], $S^{1} \tilde{\times} S^{3} \sharp K \sharp\left(\sharp^{r} S^{2} \times S^{2}\right)$ is homeomorphic to $S^{1} \tilde{\times} S^{3} \sharp\left(\sharp^{r+11} S^{2} \times S^{2}\right)$. Let us show that $S^{1} \tilde{\times} S^{3} \sharp K \sharp\left(\sharp^{r} S^{2} \times S^{2}\right)$ is not diffeomorphic to $\Sigma \sharp S^{1} \tilde{\times} S^{3} \sharp\left(\sharp^{r+11} S^{2} \times S^{2}\right)$ for any homotopy 4 -sphere $\Sigma$. We use the same notation as in [23]. Kreck [23] has shown that $S^{1} \tilde{\times} S^{3} \sharp K \sharp\left(\sharp^{r} S^{2} \times S^{2}\right)$ is not diffeomorphic to $S^{1} \tilde{\times} S^{3} \sharp\left(\sharp^{r+11} S^{2} \times S^{2}\right)$ by showing that they are not equivalent in $\Omega_{4}^{B} / \operatorname{Aut}(B)$. It is not difficult to see that $\Sigma^{4}$ represents zero in $\Omega_{4}^{B}$, since $\Sigma^{4}$ bounds a compact smooth contractible 5-dimensional manifold. Thus $\Sigma^{4} \sharp S^{1} \tilde{\times} S^{3} \sharp\left(\sharp^{r+11} S^{2} \times S^{2}\right)$ and $S^{1} \tilde{\times} S^{3} \sharp K \sharp\left(\sharp^{r} S^{2} \times S^{2}\right)$ are not equivalent to each other in $\Omega_{4}^{B} / \operatorname{Aut}(B)$ and hence are not diffeomorphic to each other. Hence by Theorem 3.1 we have the conclusion.

Remark 3.7. In the above examples, the manifolds are nonorientable. For orientable manifolds, we have a candidate, which is a so-called Scharlemann manifold. Let $M^{\prime}$ be a Scharlemann manifold [37]. It is known that $M^{\prime}$ is topologically $s$-cobordant and hence homeomorphic to $S^{1} \times S^{3} \sharp S^{2} \times S^{2}$ by [15]. The authors do not know if the Scharlemann manifold $M^{\prime}$ admits a special generic map into $\mathbf{R}^{3}$. However, since $M^{\prime}$ is stably parallelizable, $M^{\prime}$ does admit a smooth map $g: M^{\prime} \rightarrow \mathbf{R}^{3}$ with only fold singularities by a result of Èliašberg [10]. Therefore, it naturally arises the question from the global singularity theoretical viewpoint whether one can eliminate the indefinite fold singularities of $g$ or not. This problem is closely related to the smooth structure of the manifold. If we can prove that $M^{\prime}$ does not admit any special generic map into $\mathbf{R}^{3}$, then we can show that $M^{\prime}$ is not 
diffeomorphic to $S^{1} \times S^{3} \sharp S^{2} \times S^{2}$. (See also [36].) Note that $M^{\prime} \sharp S^{2} \times S^{2}$ (resp. $M^{\prime} \sharp S^{2} \tilde{\times} S^{2}$ ) does admit a special generic map into $\mathbf{R}^{3}$, since it is diffeomorphic to $S^{1} \times S^{3} \sharp\left(\sharp^{2} S^{2} \times S^{2}\right)$ (resp. $\left.S^{1} \times S^{3} \sharp S^{2} \times S^{2} \sharp S^{2} \tilde{\times} S^{2}\right)$ [13].

\section{Fundamental groups of the source manifolds.}

Let $M$ be a closed $n$-dimensional manifold $(n \geq 4)$ which admits a special generic map $f: M \rightarrow \mathbf{R}^{3}$. In this section, we list up some results about the fundamental group of $M$, using results of 3-manifold groups. Recall that the quotient space $W_{f}$ is a compact orientable 3-dimensional manifold with fundamental group isomorphic to $\pi_{1}(M)$ (for example, see [31]).

There have been a lot of important results in the study of 3-manifold groups. For example, refer to [19], [11, Theorem 3.3], [41, Theorem A] [12], [26, §4] etc. Using these results, we can obtain a lot of properties of the fundamental groups of those manifolds which admit special generic maps into $\mathbf{R}^{3}$. For example, we can deduce the following result.

Proposition 4.1. Suppose that a closed $n$-dimensional manifold $M(n \geq 4)$ admits a special generic map into $\mathbf{R}^{3}$ and let $G$ be a subgroup of $\pi_{1}(M)$ which is a finitely generated abelian group. Then $G$ is isomorphic to $\mathbf{Z}, \mathbf{Z} \oplus \mathbf{Z}, \mathbf{Z} \oplus$ $\mathbf{Z} \oplus \mathbf{Z}$, or $\mathbf{Z} / p \mathbf{Z}$ for some positive integer $p$.

The above proposition implies, for example, that the $n$-dimensional torus $T^{n}(n \geq 4)$ does not admit any special generic map into $\mathbf{R}^{3}$.

Remark 4.2. For a special generic map $f: M \rightarrow \mathbf{R}^{p}$ of an $n$-dimensional closed manifold $M$ into $\mathbf{R}^{p}$ with $n>p \geq 4$, there is no restriction on the fundamental group of $M$. In fact, for every pair $(n, p)$ with $n>p \geq 4$ and every finitely presentable group $\pi$, there exists a closed $n$-dimensional manifold $M$ with fundamental group isomorphic to $\pi$ which admits a special generic map into $\mathbf{R}^{p}$. This is proved by constructing a compact $p$-dimensional manifold $W$ immersed in $\mathbf{R}^{p}$ with fundamental group isomorphic to $\pi$ and then by constructing a special generic map whose quotient space is diffeomorphic to $W$.

In the following, we shall see that the fundamental group of the source manifold often clarifies its diffeomorphism type.

Theorem 4.3. Let $f: M \rightarrow \mathbf{R}^{3}$ be a special generic map of a closed $n$-dimensional manifold with $n=4,5,6$ or 7 . Suppose that $\pi_{1}(M)$ has a subgroup $G$ of finite index which is isomorphic to $\pi_{1}(F)$ for some closed surface $F$ not homeomorphic to $S^{2}$ or $\mathbf{R} P^{2}$. Then $M$ is diffeomorphic to

$$
M_{0} \sharp\left(M_{1} \sharp \cdots \sharp M_{r}\right) \sharp \Sigma^{n},
$$


where $M_{0}$ is a smooth $S^{n-2}$-bundle over a closed surface, $M_{1}, \ldots, M_{r}$ are smooth $S^{n-2}$-bundles over $S^{2}$ and $\Sigma^{n}$ is a homotopy $n$-sphere.

Proof. By [19, Theorem 10.6], we see that $W_{f}$ is diffeomorphic to $F_{1} \times$ $I \sharp\left(\sharp^{r} B^{3}\right) \sharp \Sigma^{3}$ or $W_{1} \sharp\left(\sharp^{r} B^{3}\right) \sharp \Sigma^{3}$, where $F_{1}$ is a closed surface, $I=[0,1], B^{3}$ is the 3-dimensional disk, $\Sigma^{3}$ is a homotopy 3 -sphere and $W_{1}$ is a twisted $I$ bundle over a closed surface $F_{1}$. Thus $W_{f}$ is diffeomorphic to $F_{1} \times I \emptyset\left(\natural^{r} S^{2} \times\right.$ $I) \natural \Delta$ or $W_{1} \natural\left(\natural^{r} S^{2} \times I\right) \natural \Delta$, where $\Delta=\Sigma-$ Int $D^{3}$. Thus, using an argument similar to that used in the proof of Theorem 2.1, we see that $M$ is diffeomorphic to $M_{0} \sharp\left(M_{1} \sharp \cdots \sharp M_{r}\right) \sharp \Sigma^{n}$, where $M_{0}$ is a smooth $S^{n-2}$-bundle over $F_{1}$, the manifolds $M_{1}, \ldots, M_{r}$ are smooth $S^{n-2}$-bundles over $S^{2}$ and $\Sigma^{n}=\partial\left(\Delta \times D^{n-2}\right)$. This completes the proof.

Compare the above theorem with [8] when $n=4$.

Remark 4.4. The above theorem is valid also for all $n \geq 8$ with $\Theta_{n-1}=0$. Furthermore, we have $\Sigma^{n} \cong S^{n}$ for all $n \geq 5$. These remarks are valid also for Propositions 4.5, 4.6 and Theorem 4.7.

Proposition 4.5. Suppose that $f: M \rightarrow \mathbf{R}^{3}$ is a special generic map of a closed $n$-dimensional manifold with $n=4,5,6$ or 7 and that $S(f)$ contains a component not homeomorphic to $S^{2}$. If $\pi_{1}(M)$ is infinite and is a nontrivial direct product, then $M$ is diffeomorphic to $M_{0} \sharp\left(M_{1} \sharp \cdots \sharp M_{r}\right) \sharp \Sigma^{n}$, where $M_{0}$ is a smooth $N$-bundle over $S^{1}$ for some closed $(n-1)$-dimensional manifold $N$ admitting a special generic map into $\mathbf{R}^{2}$, the manifolds $M_{1}, \ldots, M_{r}$ are smooth $S^{n-2}$-bundles over $S^{2}$ and $\Sigma^{n}$ is a homotopy $n$-sphere.

Proof. By [19, Chapter 11], we see that $W_{f}$ is diffeomorphic to $\left(F \times S^{1}\right) \sharp$ $\left(\sharp^{r} B^{3}\right) \sharp \Sigma^{3}$, where $F$ is a compact orientable surface and $\Sigma$ is a homotopy 3 -sphere. Since $\partial W_{f}$ contains a component not homeomorphic to $S^{2}$ by our hypothesis, $F$ has nonempty boundary. Thus $W_{f}$ is diffeomorphic to $\left(F \times S^{1}\right) \natural\left(\natural^{r} S^{2} \times I\right) \natural \Delta$, where $\Delta=\Sigma-\operatorname{Int} D^{3}$. Hence $M$ is diffeomorphic to $M_{0} \sharp\left(M_{1} \sharp \cdots \sharp M_{r}\right) \sharp \Sigma^{n}$, where $M_{0}$ is a smooth $N$-bundle over $S^{1}$ for some closed $(n-1)$-dimensional manifold $N$ admitting a special generic map into $\mathbf{R}^{2}$ with the quotient space diffeomorphic to $F$, the manifolds $M_{1}, \ldots, M_{r}$ are smooth $S^{n-2}$-bundles over $S^{2}$ and $\Sigma^{n}=\partial\left(\Delta \times D^{n-2}\right)$. This completes the proof.

Recall that those closed $(n-1)$-dimensional manifolds $N$ which admit a special generic map into $\mathbf{R}^{2}$ are classified in [4] and [31] (see also [30]). 
An argument similar to the above shows that the product of closed connected orientable surfaces $\Sigma_{g}$ and $\Sigma_{g^{\prime}}$ of genera $g$ and $g^{\prime}$ respectively admits a special generic map into $\mathbf{R}^{3}$ if and only if $g g^{\prime}=0$.

Proposition 4.6. Let $f: M \rightarrow \mathbf{R}^{3}$ be a special generic map of a closed $n$-dimensional manifold with $n=4,5,6$ or 7 . Suppose that $\pi_{1}(M)$ is infinite and that its center is a nontrivial, finitely generated noncyclic group. Then $M$ is diffeomorphic to $M_{0} \sharp\left(M_{1} \sharp \cdots \sharp M_{r}\right) \sharp \Sigma^{n}$, where $M_{0}$ is a smooth $S^{n-2}$ bundle over $T^{2}$, the manifolds $M_{1}, \ldots, M_{r}$ are smooth $S^{n-2}$-bundles over $S^{2}$ and $\Sigma^{n}$ is a homotopy $n$-sphere.

Proof. By [19, Theorem 12.10], we see that $W_{f}$ is diffeomorphic to $T^{2} \times$ $I \sharp\left(\sharp^{r} B^{3}\right) \sharp \Sigma$ for some homotopy 3 -sphere $\Sigma$. Then the conclusion follows from an argument similar to the above.

Theorem 4.7. Let $f: M \rightarrow \mathbf{R}^{3}$ be a special generic map of a closed $n$-dimensional manifold with $n=4,5,6$ or 7 and that $S(f)$ contains a component not homeomorphic to $S^{2}$. If $\pi_{1}(M)$ is infinite and solvable, then $M$ is diffeomorphic to $M_{0} \sharp\left(M_{1} \sharp \cdots \sharp M_{r}\right) \sharp \Sigma^{n}$, where $M_{1}, \ldots, M_{r}$ are smooth $S^{n-2}$ bundles over $S^{2}, \Sigma^{n}$ is a homotopy $n$-sphere and $M_{0}$ is a smooth $S^{n-1}$-bundle over $S^{1}$, a smooth $S^{n-2}$-bundle over $T^{2}$ or a smooth $S^{n-2}$-bundle over the Klein bottle.

Proof. By [12], we see that $W_{f}$ is diffeomorphic to $W_{1} \sharp\left(\sharp^{r} B^{3}\right) \sharp \Sigma$, where $W_{1}$ is $S^{1} \times D^{2}, T^{2} \times I$ or the twisted $I$-bundle over the Klein bottle. Then the conclusion follows from an argument similar to the above.

The deficiency of a finitely presentable group $\pi$ is the maximum over all differences $g-r$, where $g$ and $r$ are the numbers of generators and relations respectively of a presentation of $\pi$ (see, for example, [11]).

Proposition 4.8. Let $f: M \rightarrow \mathbf{R}^{3}$ be a special generic map of a closed $n$ dimensional manifold with $n \geq 4$. Let $W_{f}$ denote the quotient space, which is a compact 3-manifold, and let

$$
W_{f}=W_{1} \sharp \cdots \sharp W_{r}
$$

be its prime decomposition. Then the deficiency of $\pi_{1}(M)$ is equal to $s\left(W_{f}\right)+$ $t\left(W_{f}\right)-\chi\left(W_{f}\right)$, where $s\left(W_{f}\right)$ is the number of prime factors $W_{i}$ with nonempty boundary, $t\left(W_{f}\right)$ is the number of prime factors $W_{i}$ homeomorphic to $S^{1} \times S^{2}$ and $\chi$ denotes the Euler characteristic. In particular, the integer $s\left(W_{f}\right)+t\left(W_{f}\right)-\chi(S(f)) / 2$ is an invariant of $M$ when $n$ is odd, and the integer $s\left(W_{f}\right)+t\left(W_{f}\right)$ is an invariant of $M$ when $n$ is even. 
Proof. This is a direct consequence of [28, Theorem 6.3] and [31, Proposition 3.9].

Corollary 4.9. Let $f: M \rightarrow \mathbf{R}^{3}$ be a special generic map of a closed $n$ dimensional manifold with $n \geq 4$ and with $\pi_{1}(M)$ a free group of rank $r$. Then we have $s\left(W_{f}\right)+t\left(W_{f}\right)-\chi(S(f)) / 2=r$. In particular, when $n$ is even, we have $s\left(W_{f}\right)+t\left(W_{f}\right)=\chi(M) / 2+r$.

\section{Number of sphere components in the singular set and $L^{2}$-Betti numbers.}

It is known that the singular set of a generic map of a manifold $M$ with $\operatorname{dim} M \geq 3$ into $\mathbf{R}^{3}$ is a smooth 2-dimensional submanifold of $M$. Given such a manifold $M$, it is difficult to say something general about the topological structure of those 2-dimensional submanifolds which arise as the singular set of a generic map into $\mathbf{R}^{3}$ on $M$ (e.g. the number of connected components, their orientability, the genus of each component, etc.), since it heavily depends on the choice of the generic map (for example, see the next section). However, in the case of special generic maps into $\mathbf{R}^{3}$, the authors have obtained several results concerning the relationship between the Betti numbers of the source manifold and the topology of the singular set (see [31], [35]). They are some inequalities concerning the Betti numbers of the source manifold and the singular set and they hold only when the fundamental group of the source manifold is very "close to" being trivial (see [31], [32], [34], [35]). In this section, we consider the $L^{2}$-Betti numbers of the source manifold in stead of the ordinary Betti numbers and obtain some equalities concerning the $L^{2}$-Betti numbers of the source manifold and the number of sphere components in the singular set of a special generic map. For the definition of the $L^{2}$-Betti numbers, see [27] or [17], for example. Recall that the $L^{2}$-Betti numbers are homotopy invariants for finite $\mathrm{CW}$ complexes, which are defined by using the Hilbert spaces arising from the chain complex of the universal covering space.

Theorem 5.1. Let $f: M \rightarrow \mathbf{R}^{3}$ be a special generic map of a closed connected $n$-dimensional manifold with $n \geq 4$. Suppose that $\pi_{1}(M)$ is indecomposable with respect to free products and that $S(f)$ contains a component not homeomorphic to $S^{2}$. Then we have

$$
\begin{aligned}
& b_{0}^{(2)}(M)=0, \\
& b_{1}^{(2)}(M)=-\frac{1}{2} \chi(S(f))+\sharp\left\{S \in \pi_{0}(S(f)): S \cong S^{2}\right\},
\end{aligned}
$$




$$
\begin{aligned}
& b_{2}^{(2)}(M)= \begin{cases}2 \sharp\left\{S \in \pi_{0}(S(f)): S \cong S^{2}\right\} & \text { if } n=4, \\
\sharp\left\{S \in \pi_{0}(S(f)): S \cong S^{2}\right\} & \text { if } n \geq 5,\end{cases} \\
& b_{p}^{(2)}(M)=0 \quad\left(3 \leq{ }^{\forall} p \leq n-3\right),
\end{aligned}
$$

where $\sharp\left\{S \in \pi_{0}(S(f)): S \cong S^{2}\right\}$ denotes the number of components $S$ of $S(f)$ diffeomorphic to $S^{2}$ and $b_{i}^{(2)}(M)$ denotes the $i$-th $L^{2}$-Betti number of $M$.

For the proof of this theorem, we need the following.

Lemma 5.2. Let $f: M \rightarrow N$ be a special generic map of a closed $n$ dimensional manifold into a p-dimensional manifold. If $p \leq(n+1) / 2$, then $W_{f}$ has the homotopy type of a finite $C W$ complex which is obtained by attaching cells of dimension greater than or equal to $n-p+2$ to $M$.

Proof. By [31], $M$ is homeomorphic to the boundary of a topological $D^{n-p+1}$ bundle $E$ over $W_{f}$. Since $W_{f}$ is a compact smooth manifold, it has a structure of a finite CW complex. Furthermore, we may assume that it has a unique 0 -cell and that it lies in $\partial W_{f}$. Since $W_{f}$ has the homotopy type of a finite CW complex of dimension $p-1$ and we have $p-1 \leq n-p$ by our assumption, we see that there exists a section for the topological $S^{n-p}$-bundle associated with $E$. Then, using this section together with the CW complex structure of $W_{f}$, we can first construct a finite CW complex structure for $\partial E$, which is homeomorphic to $M$. Then we can construct a finite CW complex structure for $E$ by attaching cells of dimension greater than or equal to $n-p+2$ to $\partial E$. Since $E$ has the same homotopy type as $W_{f}$, we have the conclusion.

Corollary 5.3. Let $f: M \rightarrow N$ be a special generic map of a closed $n$ dimensional manifold into a $p$-dimensional manifold. If $p \leq(n+1) / 2$, then $b_{i}^{(2)}\left(W_{f}\right)=b_{i}^{(2)}(M)$ for all $i$ with $i \leq n-p$.

Proof of Theorem 5.1. It is easy to show that $W_{f}$ is diffeomorphic to $W^{\prime} \sharp\left(\sharp^{r} B^{3}\right) \sharp \Sigma$, where $W^{\prime}$ is an irreducible sufficiently large 3-manifold (i.e. Haken) and $\Sigma$ is a homotopy 3 -sphere. Since $b_{i}^{(2)}\left(W_{f}\right)=b_{i}^{(2)}\left(W^{\prime} \sharp\left(\sharp^{r} B^{3}\right)\right)$ for all $i$, the conclusion follows from [27, Lemma 6.4] and Corollary 5.3 when $n \geq 5$. When $n=4$, we have the result for $b_{0}^{(2)}(M)$ and $b_{1}^{(2)}(M)$ since these numbers depend only on the fundamental group (see [28, Lemma 1.2]). We can obtain the result for $b_{2}^{(2)}(M)$ by using the facts that $\chi(M)=2 b_{0}^{(2)}(M)-$ $2 b_{1}^{(2)}(M)+b_{2}^{(2)}(M)$ and that $\chi(M)=\chi(S(f))([\mathbf{1 6}],[31],[35])$. This completes the proof. 
Remark 5.4. In Theorem 5.1, the condition that $S(f)$ contains a component not homeomorphic to $S^{2}$ is assured when the Euler characteristic of the source manifold is nonpositive and $n$ is even, for example. This follows from the fact that the Euler characteristic of $M$ coincides with that of the singular set if $n$ is even [16], [31], [35].

Remark 5.5. Theorem 5.1 shows that the number of sphere components of the singular set is independent of the special generic map.

Compare Theorem 5.1 with the results obtained in [17].

\section{Realizing arbitrary two surfaces as singular sets.}

Recall that for a special generic map $f: M \rightarrow \mathbf{R}^{3}$ of a closed 4-dimensional manifold, the Euler characteristic of the singular set $S(f)$ of $f$ is equal to that of $M$ (see [16], [31, Proposition 3.5] and [35, Corollary 2.7]).

Theorem 6.1. Let $S_{1}$ and $S_{2}$ be closed (not necessarily connected) orientable surfaces with the same Euler characteristic. Then there exist a closed connected orientable 4-manifold $M$ and special generic maps $f_{i}: M \rightarrow \mathbf{R}^{3}$ $(i=1,2)$ such that the singular set $S\left(f_{i}\right)$ of $f_{i}$ is diffeomorphic to $S_{i}$.

In the following, for a compact $n$-dimensional manifold $N$ with nonempty boundary, we define the spun manifold $\operatorname{spun} N$ of $N$ to be the closed $(n+1)$ dimensional manifold $\partial\left(N \times D^{2}\right)$. Note that spun manifolds are usually defined for closed manifolds. More precisely, for a closed $n$-dimensional manifold $N$, spun $N$ is usually defined to be $\partial\left(N^{\circ} \times D^{2}\right)$, where $N^{\circ}=N-$ Int $D^{n}$ (see [40], for example). Here we define the spun manifold in a slightly different manner.

Proof of Theorem 6.1. Suppose that $S_{1}$ is diffeomorphic to $\Sigma_{g_{1}} \cup \cdots \cup \Sigma_{g_{k}}$ and that $S_{2}$ is diffeomorphic to $\Sigma_{h_{1}} \cup \cdots \cup \Sigma_{h_{l}}$, where $\Sigma_{g}$ denotes a closed connected orientable surface of genus $g$. We may assume that $l \geq k(\geq 1)$. Note that $\chi\left(S_{1}\right)=\chi\left(S_{2}\right)$ is equivalent to

$$
\sum_{i=1}^{k}\left(1-g_{i}\right)=\sum_{j=1}^{l}\left(1-h_{j}\right),
$$

where $\chi$ denotes the Euler characteristic. Set

$$
L_{1}=\left(\natural^{g_{1}} S^{1} \times D^{2}\right) \sharp \cdots \sharp\left(\natural^{g_{k}} S^{1} \times D^{2}\right) \sharp\left(\sharp^{l-k} S^{1} \times S^{2}\right)
$$

and

$$
L_{2}=\left(\natural^{h_{1}} S^{1} \times D^{2}\right) \sharp \cdots \sharp\left(\natural^{h_{l}} S^{1} \times D^{2}\right) .
$$


Here, when $g=0$, we regard $\natural^{g} S^{1} \times D^{2}=D^{3}$ and $\sharp^{g} S^{1} \times S^{2}=S^{3}$. Note that $\partial L_{1} \cong S_{1}$ and $\partial L_{2} \cong S_{2}$. Then we have

$$
\begin{gathered}
L_{1} \cong\left(\natural^{g_{1}} S^{1} \times D^{2}\right) \downarrow\left(\left(\natural^{g_{2}} S^{1} \times D^{2}\right)-\operatorname{Int} D^{3}\right) \downarrow \cdots \downarrow\left(\left(\natural^{g_{k}} S^{1} \times D^{2}\right)-\operatorname{Int} D^{3}\right) \\
\natural\left(\natural^{l-k}\left(S^{1} \times S^{2}-\operatorname{Int} D^{3}\right)\right)
\end{gathered}
$$

and

$$
\left(\natural^{g} S^{1} \times D^{2}\right)-\operatorname{Int} D^{3} \cong\left(S^{2} \times I\right) \natural\left(\natural^{g} S^{1} \times D^{2}\right) .
$$

Then we have $\operatorname{spun}\left(L_{1}\right)=\partial\left(L_{1} \times D^{2}\right) \cong\left(\sharp^{G} S^{1} \times S^{3}\right) \sharp\left(\sharp^{k-1} S^{2} \times S^{2}\right) \sharp\left(\sharp^{l-k} S^{2} \times\right.$ $\left.S^{2}\right) \sharp\left(\sharp^{l-k} S^{1} \times S^{3}\right) \cong\left(\sharp^{G+l-k} S^{1} \times S^{3}\right) \sharp\left(\sharp^{l-1} S^{2} \times S^{2}\right) \cong \operatorname{spun}\left(L_{2}\right)$, where $G=$ $\sum_{i=1}^{k} g_{i}$ (see the equation (1)). Thus, putting $M=\operatorname{spun}\left(L_{1}\right) \cong \operatorname{spun}\left(L_{2}\right)$, we see that there exist special generic maps $f_{1}: M \rightarrow \mathbf{R}^{3}$ and $f_{2}: M \rightarrow \mathbf{R}^{3}$ such that $W_{f_{1}}=L_{1}$ and $W_{f_{2}}=L_{2}$. (Note that $L_{1}$ and $L_{2}$ are parallelizable. See [31, Proposition 2.1].) Then $S\left(f_{1}\right) \cong \partial L_{1} \cong S_{1}$ and $S\left(f_{2}\right) \cong \partial L_{2} \cong S_{2}$. This completes the proof.

Remark 6.2. By a similar argument, we can show that for any finite set of closed orientable surfaces $S_{1}, \ldots, S_{r}$ with the same Euler characteristic, there exist a closed connected 4-manifold $M$ and special generic maps $f_{i}: M \rightarrow \mathbf{R}^{3}$ $(i=1, \ldots, r)$ such that the singular set $S\left(f_{i}\right)$ of $f_{i}$ is diffeomorphic to $S_{i}$.

Remark 6.3. In Remark 5.5, we have seen that if $\pi_{1}(M)$ is indecomposable with respect to free products, then the number of sphere components is independent of special generic maps as long as the singular set contains a component not homeomorphic to $S^{2}$. Thus, when $\pi_{1}(M)$ is indecomposable, we do not have results like Theorem 6.1. Note that the manifold constructed in the proof of Theorem 6.1 has free fundamental groups which are decomposable in general.

Definition 6.4. Let $M$ be a closed connected $n$-dimensional manifold and $p$ an integer with $n>p>0$. We denote by $q(M, p)$ the number of quasiequivalence classes of special generic maps of $M$ into $\mathbf{R}^{p}$ (see [32, Definition 4.1]).

Recall that for a closed connected 3 -manifold $M$, the number $q(M, 2)$ is always finite by $[4]$.

Theorem 6.5. For a positive integer $p$, let $l(p)$ denote the number of homeomorphism classes of 3 -dimensional lens spaces with $\pi_{1} \cong \mathbf{Z} / p \mathbf{Z}$. Then for $M=\operatorname{spun}\left(L(p, 1)-\operatorname{Int} D^{3}\right)$, we have $q(M, 3) \geq l(p)$. 
Proof. Let $L(p, q)$ be the lens space of type $(p, q)$. Then by [29], [21], the diffeomorphism type of $M=\operatorname{spun}\left(L(p, q)-\operatorname{Int} D^{3}\right)$ does not depend on $q$. Thus, by using techniques of [31], we can construct a special generic map $f: M \rightarrow \mathbf{R}^{3}$ such that $W_{f}$ is diffeomorphic to $L(p, q)-\operatorname{Int} D^{3}$. Since the homeomorphism class of $W_{f}$ is an invariant of the quasi-equivalence class of $f$, we have the conclusion. This completes the proof.

Note that in Theorem 6.5, the singular set is always diffeomorphic to the 2-sphere by [31, Corollary 3.14, Proposition 3.15] and [35, Theorem B].

Problem 6.6. Does there exist a closed connected 4-manifold $M$ such that $q(M, 3)=\infty$ ? Note that $q\left(S^{5}, 4\right)=\infty$ by virtue of $[31, \S 4]$.

For further related problems, see $[32, \S 5]$.

\section{References}

[1] S. Akbulut, A fake 4-manifold, Contemporary Math., 35 (1984), 75-141.

[2] _ On fake $S^{3} \tilde{\times} S^{1} \sharp S^{2} \times S^{2}$, Contemporary Math., 44 (1985), 281-286.

[3] Constructing a fake 4-manifold by Gluck construction to a standard 4manifold, Topology, 27 (1988), 239-243.

[4] O. Burlet and G. de Rham, Sur certaines applications génériques d'une variété close à trois dimensions dans le plan, Enseign. Math., 20 (1974), 275-292.

[5] S.E. Cappell, On connected sums of manifolds, Topology, 13 (1974), 395-400.

[6] _ A splitting theorem for manifolds, Inv. Math., 33 (1976), 69-170.

[7] S.E. Cappell and J.L. Shaneson, Some new four-manifolds, Ann. of Math., 104 (1976), 61-72.

[8] A. Cavicchioli, F. Hegenbarth and D. Repovš, On the stable classification of certain 4-manifolds, Bull. Austral. Math. Soc., 52 (1995), 385-398.

[9] J. Cerf, Sur les difféomorphismes de la sphère de dimension trois $\left(\Gamma_{4}=0\right)$, Lecture Notes in Math., Vol. 53, Springer, 1968.

[10] J.M. Èliašberg, Surgery of singularities of smooth mappings, Math. USSR-Izv., 6 (1972), 1302-1326.

[11] D.B.A. Epstein, Finite presentations of groups and 3-manifolds, Quart. J. Math., 12 (1961), 205-212.

[12] B. Evans and L. Moser, Solvable fundamental groups of compact 3-manifolds, Trans. Amer. Math. Soc., 168 (1972), 189-210.

[13] R. Fintushel and P.S. Pao, Identification of certain 4-manifolds with group actions, Proc. Amer. Math. Soc., 67 (1977), 344-350.

[14] R. Fintushel and R.J. Stern, Another construction of an exotic $S^{1} \times S^{3} \sharp S^{2} \times S^{2}$, Contemporary Math., 35 (1984), 269-275. 
[15] M.H. Freedman, The disk theorem for four-dimensional manifolds, Proc. Int. Congress of Math., August 16-24, 1983, Warszawa, pp. 647-663.

[16] T. Fukuda, Topology of folds, cusps and Morin singularities, in 'A Fete of Topology' (eds. Y. Matsumoto, T. Mizutani and S. Morita), Academic Press, New York, (1987), 331-353.

[17] Y. Hara, Special generic maps and $L^{2}$-Betti numbers, to appear in Osaka J. Math.

[18] A.E. Hatcher, A proof of the Smale conjecture, Diff $\left(S^{3}\right) \simeq O(4)$, Ann. of Math., 117 (1983), 553-607.

[19] J. Hempel, 3-Manifolds, Ann. of Math. Stud., Vol. 86, Princeton Univ. Press, Princeton, N.J., 1976.

[20] J.T. Hiratuka, Aplicações genéricas especiais de uma variedade fechada de dimensão $n+1$ no $\mathbf{R}^{n}$, Master Thesis, University of São Paulo, 1992.

[21] Z. Iwase, Good torus fibrations with twin singular fibers, Japan. J. Math., 10 (1984), 321-352.

[22] A. Kawauchi, Splitting a 4-manifold with infinite cyclic fundamental group, Osaka J. Math., 31 (1994), 489-495.

[23] M. Kreck, Some closed 4-manifolds with exotic differentiable structure, Lecture Notes in Math., Vol. 1051, Springer, (1984), 246-262.

[24] M. Kreck, W. Lück and P. Teichner, Stable prime decompositions of four-manifolds, in 'Prospects in topology', Proc. of a Conference in Honor of W. Browder, ed. by F. Quinn, Ann. of Math. Stud., Vol. 138, Princeton Univ. Press, Princeton, N.J., 1995.

[25] Counterexamples to the Kneser conjecture in dimension four, Comment. Math. Helv., 70 (1995), 423-433.

[26] R. Lee, Semicharacteristic classes, Topology, 12 (1973), 183-199.

[27] J. Lott and W. Lück, $L^{2}$-topological invariants of 3-manifolds, Inv. Math., 120 (1995), 15-60.

[28] W. Lück, L2-Betti numbers of mapping tori and groups, Topology, 33 (1994), 203214 .

[29] P.S. Pao, The topological structure of 4-manifolds with effective torus actions, I, Trans. Amer. Math. Soc., 227 (1977), 279-317.

[30] P. Porto and Y.K.S. Furuya, On special generic maps from a closed manifold into the plane, Topology Appl., 35 (1990), 41-52.

[31] O. Saeki, Topology of special generic maps of manifolds into Euclidean spaces, Topology Appl., 49 (1993), 265-293.

[32] Topology of special generic maps into $\mathbf{R}^{3}$, in 'Workshop on Real and Complex Singularities', Matemática Contemporânea, 5 (1993), 161-186.

[33] O. Saeki and K. Sakuma, Elimination of singularities: Thom polynomial and beyond, to appear in Proc. in Honour of C.T.C. Wall's 60-th Birthday, Cambridge Univ. Press.

[34] K. Sakuma, On special generic maps of simply connected 2 -manifolds into $\mathbf{R}^{3}$, Topology Appl., 50 (1993), 249-261.

[35] _ On the topology of simple fold maps, Tokyo J. Math., 17 (1994), 21-31.

[36] Y. Sato, Scharlemann's 4-manifolds and smooth 2-knots in $S^{2} \times S^{2}$, Proc. Amer. Math. Soc., 121 (1994), 1289-1294. 
[37] M. Scharlemann, Constructing strange manifolds with the dodecahedral space, Duke Math. J., 43 (1976), 33-40.

[38] S. Smale, Diffeomorphisms of the 2-sphere, Proc. Amer. Math. Soc., 10 (1959), 621-626.

[39] S. Stolz, Exotic structures on 4-manifolds detected by spectral invariants, Inv. Math., 94 (1988), 147-162.

[40] A.I. Suciu, The oriented homotopy type of spun 3-manifolds, Pacific J. Math., 131 (1988), 393-399.

[41] C. Thomas, Nilpotent groups and compact 3-manifolds, Proc. Camb. Phil. Soc., 64 (1968), 303-306.

Received June 30, 1996 and revised April 2, 1997. The first author was partially supported by Grant-in-Aid for Encouragement of Young Scientists (No. 08740057), Ministry of Education, Science and Culture, Japan. The second author was partially supported by Grant-in-Aid for Encouragement of Young Scientists (No. 08874004), Ministry of Education, Science and Culture, Japan.

\author{
HiROSHIMA UNIVERSITY \\ HigASHI-HIROSHIMA 739-8526 \\ JAPAN \\ E-mail address: saeki@top2.math.sci.hiroshima-u.ac.jp \\ AND \\ Kochi National College of Technology \\ NANKOKU-CITY, KOCHI 783 \\ JAPAN \\ E-mail address: sakuma@ge.kochi-ct.ac.jp \\ Current address of K. Sakuma: \\ KINKI UNIVERSITY \\ Higashi-Osaka, Osaka 577-8502 \\ JAPAN \\ E-mail address: sakuma@math.kindai.ac.jp
}

